\title{
Strongly correlated electron systems and quantum magnetism
}

We dedicate this special issue to Andrij Shvaika and Oleg Derzhko in celebration of their 60th anniversaries. Both Andrij and Oleg have been colleagues throughout their lives, as illustrated in the photos, one showing them during their primary school years and the other showing them at a recent conference held in Lviv in 2019. Andrij and Oleg have worked at the same Institute for decades and are also both Associate Editors of the Condensed Matter Physics journal. It is fitting to celebrate the two of them together in this special issue. The selected contributions cover a wide range of topics closely related to the leading scientific interests of both jubilees to acknowledge their unique and valuable contributions to science. It is our great pleasure to thank all the authors who supported this project by submitting their papers to this special issue, all the anonymous referees who carefully read and constructively reviewed them and the staff of Condensed Matter Physics who took care about the special issue at the final stage.

This special issue is focused on the recent developments in the study of strongly correlated electron systems and quantum spin systems. Of course, the research field of strongly correlated systems and quantum magnetism is too broad to be comprehensively covered and, hence, the present special issue touches only a small part of it and is by no means exhaustive. The papers collected in the special issue can be nevertheless considered as a representative collection of the current state-of-the-art, which elucidates a few challenging and highly topical issues to deal with in the field of strongly correlated electron systems and quantum magnetism. More importantly, they have been prepared as a tribute to our dear colleagues to celebrate their scientific careers reaching this important milestone.

Strongly correlated systems usually do not yield to exact solutions. There are a few celebrated exceptions often on simplified models. In other cases, one is often forced to solve the problems numerically; even there, the solutions themselves are often approximate. The present issue includes the work that examines a microscopic model for high-temperature superconductivity based on a microscopic Coulomb plus electron-phonon coupling model [1]. Andrij has worked extensively on the Falicov-Kimball model, which is one of the simplified models. In this issue we have two contributions: one examines the problem of exciton formation [2] and the other examines the interplay between on-site and extended interactions [3]. Andrij has also worked on correlated hopping, which is another one of these simplified models. We have a contribution here as well, examining both orbital degeneracy and correlated hopping [4]. We have an article investigating quantum computing, producing a so-called Schrödinger cat state on an IBM quantum computer [5]. Andrij has also studied many-particle susceptibilities in his career, in this issue we include a pedagogical introduction to deriving dynamical two-particle susceptibilities working primarily with Matsubara frequencies and functional derivatives [6].

For a long time quantum spin chains are thought of as foremost cornerstones of the quantum magnetism, which are also one of the main scientific domains of Oleg. In spite of significant achievements reported in the ground-breaking papers by Bethe, Lieb-Schultz-Mattis, Haldane, Affleck-Kennedy-LiebTasaki and others, there are still a lot of unresolved and highly debated issues concerned with various other aspects of quantum spin chains. The present special issue, for instance, clarifies the effect of bichromatic field on quantum spin chains [7] as well as the effect of three-site exchange interaction on quantum phase transitions of uniform and mixed-spin quantum chains [8]. Furthermore, the spin-1/2 quantum XY chain accounting for the magnetoelectric effect according to Katsura-Nagaosa-Balatsky mechanism represents a valuable example of the exactly solved quantum spin chain elucidating basic magnetoelectric features [9]. Experimental relevance of quantum spin chains is exemplified by the spin1/2 Ising-Heisenberg orthogonal-dimer chain [10], which brings insight into a ferrimagnetic nature of 
the intermediate plateau experimentally detected in a high-field magnetization curve of $3 \mathrm{~d}-4 \mathrm{f}$ heterobimetallic coordination polymer $\left[\mathrm{Dy}_{2} \mathrm{Cu}_{2}\right]_{n}$. The spin-1/2 Ising-Heisenberg diamond chain refined by the magnetoelastic coupling additionally brought insight into anomalous thermodynamic behavior in the vicinity of a triple coexistence point [11].

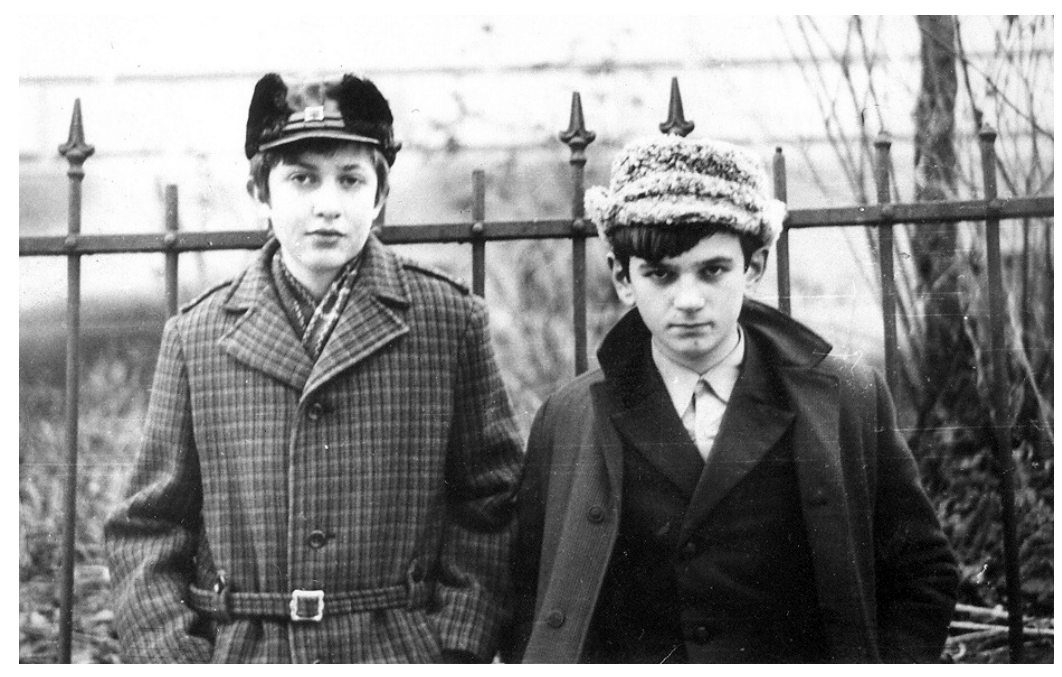

School photo of Andrij and Oleg in early 1970-ies.

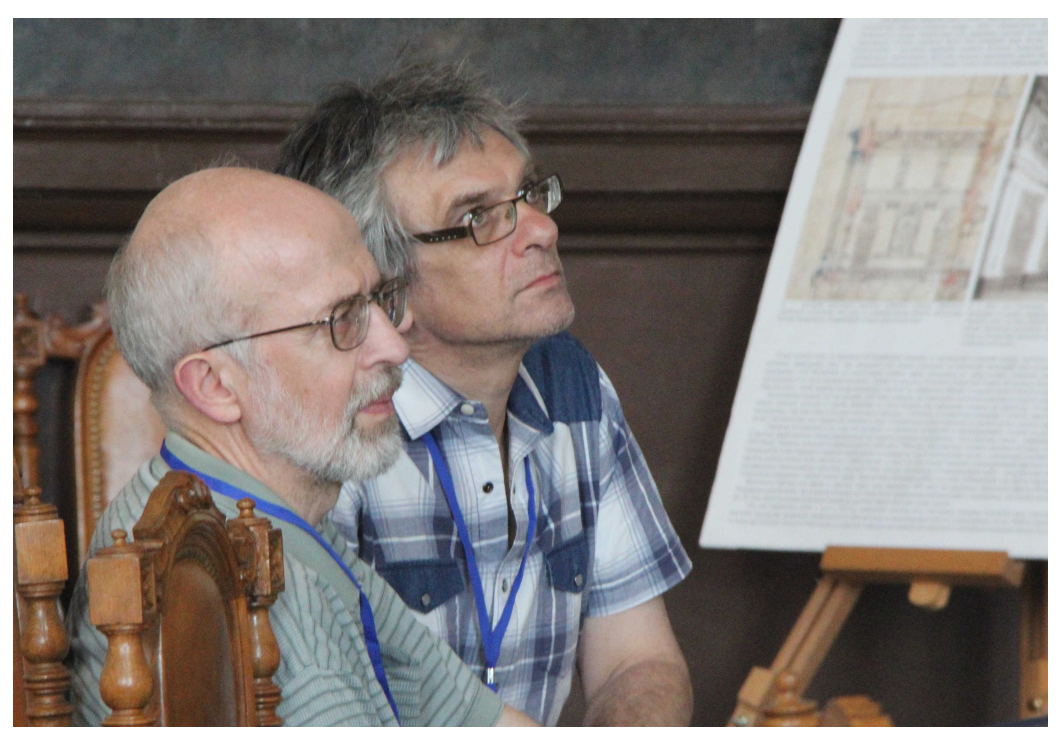

Photo of Andrij and Oleg at the 5-th Conference on Statistical Physics in Lviv 2019.

Frustrated quantum magnetism represents another important topical issue to deal with, particularly in connection with the flat-band physics, which over the past few years represents the principal subject of Oleg's studies. It has been verified in this special issue that the completely flat one-magnon band of highly frustrated kagomé antiferromagnets gives rise to macroscopically degenerate localized-magnon ground 
states, which can be exactly accounted for by a classical loop-gas description [12]. The spin-S Heisenberg sawtooth chain as another paradigmatic example of a flat-band system with macroscopically degenerate ground-state manifold may exhibit, due to massive low-lying excitations, unconventional thermodynamic properties including an enhanced magnetocaloric effect [13]. The enhanced magnetocaloric effect suitable for a low-temperature cooling has also been reported for the frustrated spin-1/2 Heisenberg cupolae, which exhibit a peculiar sequence of intermediate magnetization plateaus being absent in the semi-classical Ising limit [14]. Beside this, the fundamental question related to $S U(N) \rightarrow S U(2)$ symmetry breaking in unfrustrated quantum antiferromagnets on bipartite lattice was also discussed, particularly in [15].

As you can see, this collection illustrates both the scientific contributions of Andrij Shvaika and Oleg Derzhko and their significant impacts on the field of many-body physics. These works honor this scientific legacy. And we who know Andrij and Oleg, know how deserving both are of this distinction. We look forward to many more decades of work from these highly talented scientists.

James K. Freericks

Department of Physics, Georgetown University, 37th and O Sts. NW, Washington, DC 20057, U.S.A.

Jozef Strečka

Department of Theoretical Physics and Astrophysics, Faculty of Science, P. J. Šafárik University, Park Angelinum 9, 04001 Košice, Slovak Republic

\section{References}

1. Plakida N.M., Microscopic theory of high-temperature superconductivity in strongly correlated electronic systems, Condens. Matter Phys., 2020, 23, 43701, doi 10.5488/CMP.23.43701.

2. Farkasovsky P., DMRG study of exciton condensation in the extended Falicov-Kimball model, Condens. Matter Phys., 2020, 23, 43709, doi 10.5488/CMP.23.43709

3. Kapcia K.J., Krawczyk J., Lemański R., Extended Falicov-Kimball model at weak onsite and intersite Coulomb interactions, Condens. Matter Phys., 2020, 23, 43706, doi 10.5488/CMP.23.43706

4. Skorenkyy Yu., Kramar O., Dovhopyaty Yu., Electron-hole asymmetry in electron systems with orbital degeneracy and correlated hopping, Condens. Matter Phys., 2020, 23, 43714, doi 10.5488/CMP.23.43714

5. Kuzmak A.R., Tkachuk V.M., Preparation and study of the entanglement of the Schrödinger cat state on the ibmq-melbourne quantum computer, Condens. Matter Phys., 2020, 23, 43001, doi 10.5488/CMP.23.43001

6. Nesselrodt R.D., Freericks J.K., A pedagogical derivation of dynamical susceptibilities, Condens. Matter Phys., 2020, 23, 43703, doi $10.5488 /$ CMP.23.43703

7. Zvyagin A.A., Effect of the bi-chromatic field on the quantum spin chain with an impurity, Condens. Matter Phys., 2020, 23, 43707, doi 10.5488/CMP.23.43707

8. Ivanov N., Heisenberg spin chains with additional isotropic three-site exchange interactions, Condens. Matter Phys., 2020, 23, 43702, doi 10.5488/CMP.23.43702

9. Ohanyan V., XY-anisotropy and magnetoelectric effect in $S=1 / 2 X Y$-spin chain, Condens. Matter Phys., 2020, 23, 43704, doi $10.5488 /$ CMP.23.43704.

10. Strečka J., Gálisová L., Verkholyak T., Insights into nature of a magnetization plateau of $3 d-4 f$ coordination polymer $\left[\mathrm{Dy}_{2} \mathrm{Cu}_{2}\right]_{n}$ from a spin-1/2 Ising-Heisenberg orthogonal-dimer chain, Condens. Matter Phys., 2020, 23, 43708, doi: $10.5488 /$ CMP.23.43708

11. Ferreira N., Torrico J., de Souza S.M., Rojas O., Strečka J., Magnetoelastic properties of a spin-1/2 IsingHeisenberg diamond chain in vicinity of a triple coexistence point, Condens. Matter Phys., 2020, 23, 43713, doi $10.5488 /$ CMP.23.43713

12. Honecker A., Richter J., Schnack J., Wietek A., Loop-gas description of the localized magnon states on the kagome lattice with open boundary conditions, Condens. Matter Phys., 2020, 23, 43712, doi $10.5488 /$ CMP.23.43712

13. Richter J., Schulenburg J., Dmitriev D.V., Krivnov V.Ya., Schnack J., Thermodynamics of a quantum spin system with large residual entropy, Condens. Matter Phys., 2020, 23, 43710, doi 10.5488/CMP.23.43710

14. Karlová K., Spin-1/2 XXZ Heisenberg cupolae: magnetization process and related enhanced magnetocaloric effect, Condens. Matter Phys., 2020, 23, 43705, doi $10.5488 /$ CMP.23.43705

15. Kolezhuk A.K., Zavertanyi T.L., $S U(N) \rightarrow S U(2)$ symmetry breaking in quantum antiferromagnets, Condens. Matter Phys., 2020, 23, 43711, doi 10.5488/CMP.23.43711 